\title{
Technè
}

La science au service de l'histoire de l'art et de la préservation des biens culturels

40 | 2014

Thérapéia. Polychromie et restauration de la sculpture dans l'Antiquité

\section{Le sort de la statuaire à la fin de l'Antiquité}

The fate of statuary in Late Antiquity

\section{Béatrice Caseau}

\section{(2) OpenEdition}

1 Journals

\section{Édition électronique}

URL : https://journals.openedition.org/techne/3842

DOI : 10.4000/techne.3842

ISSN : 2534-5168

Éditeur

C2RMF

\section{Édition imprimée}

Date de publication : 26 novembre 2014

Pagination : 106-113

ISBN : 978-2-7118-6218-4

ISSN : $1254-7867$

\section{Référence électronique}

Béatrice Caseau, « Le sort de la statuaire à la fin de l'Antiquité », Technè [En ligne], 40 | 2014, mis en ligne le 24 juillet 2020, consulté le 09 février 2022. URL : http://journals.openedition.org/techne/3842 ; DOI : https://doi.org/10.4000/techne.3842

\section{(c) (i) (9)}

La revue Technè. La science au service de l'histoire de l'art et de la préservation des biens culturels est mise à disposition selon les termes de la Licence Creative Commons Attribution - Pas d'Utilisation Commerciale - Pas de Modification 4.0 International. 


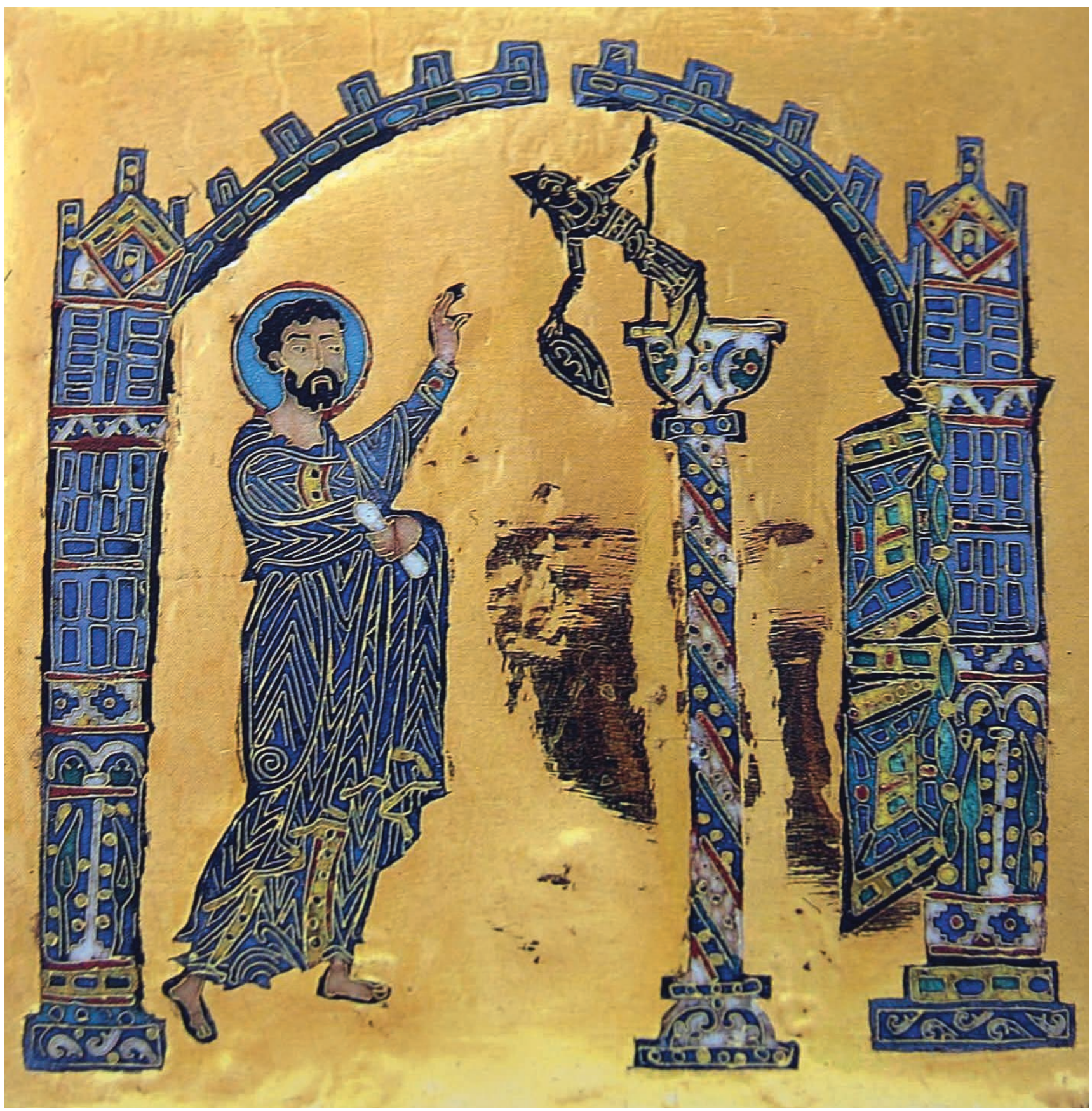

Fig. 1. Saint Marc détruit une statue

païenne. Émail de la Pala d'Oro, devant

d'autel, Saint-Marc de Venise (constitué

de diverses pièces entre le $\mathrm{x}^{\mathrm{e}}$ et le

$\mathrm{XIV}^{\mathrm{e}}$ siècle). () DR. 


\section{Béatrice Caseau Le sort de la statuaire à la fin de l'Antiquité}

The fate of statuary in Late Antiquity

Résumé. Cet article étudie la désaffection à l'égard de la statuaire antique pendant la période de la fin de l'Antiquité et le début du Moyen Âge. Il se penche sur la littérature apologétique chrétienne et les critiques que celle-ci formule à l'égard de l'attractivité que l'art confère aux statues des dieux. Le choix des métaux précieux, les formes réalistes attirent les spectateurs. Les auteurs chrétiens dénoncent les subterfuges qui permettaient de donner à penser que les statues étaient animées: articulation pour permettre le mouvement, cavité pour faire passer la voix. Les Apologistes et les auteurs d'histoires ecclésiastiques rappellent que les statues ne sont que des objets inanimés, mais il existe une littérature chrétienne, principalement hagiographique, qui croit à l'habitation des statues par les démons et souhaite leur élimination. Des légendes encore en faveur au Moyen Âge circulaient sur l'enchantement des statues. La désaffection à l'égard de la statuaire antique dépasse le rejet des idoles païennes. Des collections de statues antiques ont été mises au rebut ou bien détruites. Il faut attendre plusieurs siècles pour retrouver une valorisation de ce patrimoine.

Mots-clés. Statuaire, religions, christianisme antique, paganisme, droit romain, patristique, histoire des techniques.
Abstract. This paper studies the animosity towards Antique statuary in the period from Late Antiquity to the early Middle Ages. It examines Christian apologetic literature and the criticism formulated in regard to the attractiveness that art conferred upon statues of divinities. Spectators found the choice of precious metals and the realistic forms of the sculptures very appealing. Christian writers denounced the subterfuge used to make people think that the statues were alive: joints that moved; cavities through which a voice could speak. Apologists and ecclesiastic history writers recalled that statues were merely inanimate objects, although there was some - mainly hagiographic - Christian literature which believed that statues were inhabited by demons and should be eliminated. Legends about enchanted statues still circulated in the Middle Ages. The aversion to Antique statuary was even stronger than the rejection of pagan idols. Whole collections of ancient statues were thrown away or even destroyed. Not until several centuries later was the value of this artistic heritage reassessed.

Keywords. Statuary, religions, Early Christianity, paganism, Roman law, patristic, history of techniques.
En 382, l'empereur Théodose donnait son accord à une cité d'Osrhoène (nord-ouest de la Mésopotamie) qui avait demandé le droit de garder ouvert au public un temple afin de profiter de la beauté des statues qui y étaient conservées. À cette date déjà, l'attitude à l'égard des statues divines avait commencé à changer en même temps que la population de l'empire romano-byzantin se convertissait au christianisme et écoutait les prédicateurs comparer les dieux à des démons néfastes, les détournant du culte du vrai dieu et mettant leur âme en danger. Le christianisme avait alors pris un tournant, identifiant les statues divines avec les idoles dont le culte est dénoncé dans l'Ancien Testament et dont la destruction est vantée. En cette fin du IV siècle, les lois impériales avaient ordonné la fermeture des temples et la fin des sacrifices offerts aux divinités représentées par leurs statues, ainsi que le déplacement ou l'arrachement des statues divines recevant encore de tels sacrifices ${ }^{1}$. Mais ce virage rencontrait la résistance de siècles d'admiration pour des statues représentant une partie importante du patrimoine des cités. L’urbanisme classique reposait sur la présence d'ornements dans les espaces ouverts au public et les statues en étaient les fleurons. Si Théodose accepta la requête des habitants d'Osrhoène dans ce rescrit, c'est parce qu'il reconnut qu'il était possible de juger les statues «davantage pour leur valeur artistique que pour la divinité qu'elles représentent ${ }^{2}$. " Mais ces statues n'avaient pas perdu pour autant leur caractère sacré, du moins aux yeux d'une partie de la population demeurée païenne, et c'est précisément la beauté des statues et leur réalisme qui leur valurent la méfiance des prédicateurs chrétiens puis musulmans, ce qui entraîna finalement leur destruction ou leur mise au rebut. Si certaines furent jetées au fond des puits, ou enfouies dans des remblais, la majorité des statues de bronze fut fondue et un très grand nombre de statues de marbre a fini dans les fours à chaux du Moyen Âge. C'est ce changement culturel et esthétique qu'il convient d'expliquer. Le réalisme des statues dans lequel la polychromie a pu jouer un rôle, l'utilisation de matières pour rendre plus vif l'éclat du regard, tout ce qui était fait pour donner l'impression que la divinité

Béatrice Caseau, maître de conférence en histoire byzantine (beatrice.caseau@paris-sorbonne.fr). 
était présente dans sa statue s'est finalement retourné contre les statues des divinités, puis contre les autres statues, qu'il a alors semblé nécessaire d'éliminer.

\section{La dénonciation des artifices de l'art}

Commençons par voir comment la littérature apologétique de la période des $\mathrm{II}^{\mathrm{e}}-\mathrm{V}^{\mathrm{e}}$ siècles envisage la statuaire des dieux. Les Apologistes chrétiens dénoncent la séduction exercée par la beauté artistique et le danger d'entraînement à l'idolâtrie qu'elle comporte. L'art, explique Clément d'Alexandrie, a la force de tromper «en vous entraînant sinon à l'amour, du moins au respect, à l'adoration des statues et des peintures ${ }^{3}$ ". Pour Clément d'Alexandrie comme pour Origène, les matériaux précieux qui impressionnent les spectateurs contribuent grandement à faire croire au pouvoir des dieux. Les deux hommes ont vécu en Égypte, dans la cosmopolite cité d'Alexandrie où ils pouvaient voir des statues datant de l'antique Égypte, ainsi que celles des cultes grecs puis romains qui s'étaient implantés au fil des siècles. Des dieux de toute taille étaient représentés dans les espaces publics, les temples, les bains et les maisons. Clément d'Alexandrie évoque ainsi une statue de Sérapis qui se trouvait dans le très splendide Serapeum d'Alexandrie. Commandée par le pharaon Sésostris, elle aurait été réalisée par Bryaxis qui avait eu à sa disposition des matières premières propres à faire admirer son œuvre: «Il a employé pour son travail des matières mélangées et variées. Il avait en effet de la limaille d'or, d'argent, de cuivre, de fer, de plomb, et même d'étain; aucune des pierres précieuses d'Égypte ne lui manquait: débris de saphir, d'hématite, d'émeraude, et enfin de topaze ${ }^{4}$.» C'est un geste de vénération envers ce même dieu Sérapis qui ouvre l'Octavius, un traité de l'Africain latin chrétien Minucius Felix ( $\mathrm{II}^{\mathrm{e}}-\mathrm{III}{ }^{\mathrm{e}}$ s.) qui évoque aussi «ces gens ignorants qui se laissent abuser par les grâces de l'art, éblouir par l'éclat de l'or, fasciner par le brillant de l'argent et la blancheur de l'ivoire ${ }^{5}{ }$. Les Apologistes considèrent que l'art religieux païen induit en erreur ceux qui l'admirent. En poussant ceux qui regardent ces magnifiques sculptures à admirer tant leur taille que leur brillance, les artistes et leurs commanditaires sont responsables de la confusion qui s'introduit dans les esprits entre l'image et l'archétype.

Les Apologistes chrétiens reprochent aux statues et à ceux qui les fabriquent de faire croire, par les artifices de l'art, en la présence de la divinité dans sa statue. Le processus de fabrication des statues intéresse donc particulièrement les auteurs chrétiens, car il leur permet de rappeler que les statues ne sont que de simples productions de l'artisanat. Théophile d'Antioche (II ${ }^{\mathrm{e}}$ siècle) oppose ainsi la matière brute qui ne reçoit aucune vénération et la statue qui est traitée comme une divinité: «Voici ce qui me semble ridicule, dit-il, tailleurs de pierre, modeleurs, peintres et fondeurs façonnent, dessinent, gravent, coulent, en un mot fabriquent des dieux qui, tant qu'ils demeurent aux mains des artisans, ne sont l'objet d'aucune considération - tandis qu'après leur acquisition pour un prétendu sanctuaire ou pour une maison, non seulement ces dieux reçoivent des sacrifices de leurs acheteurs, mais on voit ceux qui les ont faits et vendus venir avec empressement, au milieu d'un déploiement de victimes et de libations, se prosterner devant eux! Ils les tiennent pour des dieux ${ }^{6}$.»

Les auteurs chrétiens insistent sur le contraste entre la dévotion que les fidèles manifestent pour les statues de culte, le soin dont ils les entourent et la brutalité nécessaire à l'artisan pour fabriquer un tel objet: «Quiconque se représentera les instruments de torture et les machines qui servent à façonner toute statue rougira de craindre une matière dont s'est joué l'artiste pour en faire un dieu ", raille Minucius Felix ${ }^{7}$. Ils ne sont pas les seuls à mettre en cause le culte des statues; certains philosophes comme Celse avaient aussi dénoncé ce qu'ils jugeaient être une forme peu raisonnée de culte. Celse qui condamne à peu près tout dans le christianisme reconnaît un mérite aux chrétiens que nous transmet Origène: «S'ils ne reconnaissent pas de divinités fabriquées de mains d'hommes, c'est qu'il n'est pas conforme à la raison que soient des dieux les objets façonnés par des artisans tout à fait vils et de caractère misérable, souvent même fabriqués par des gens injustes $^{8}$.» Dans l'Occident romain, de langue latine, les auteurs chrétiens du $\mathrm{III}^{\mathrm{e}}$ siècle scandent eux aussi ce thème. Tertullien rappelle aux païens: «Vos dieux ne sentent pas du tout ces outrages et ces affronts qu'ils subissent pendant qu'on les fabrique, pas plus qu'ils ne sentent les hommages qu'on leur rend ${ }^{9}$.» Minucius Felix ne pense pas différemment: «Un dieu de bronze ou d'argent provient bien souvent, comme cela fut le cas pour un roi d'Égypte, d'un immonde petit récipient, qui est fondu, battu à coup de maillet et façonné sur l'enclume; un dieu de pierre est taillé, sculpté et poli par un homme corrompu, et d'ailleurs il est aussi insensible aux outrages de sa naissance que plus tard aux honneurs dont l'entoure votre vénération ${ }^{10}$.»

Cependant, c'était ne pas tenir compte de l'appréciation esthétique que portaient leurs contemporains sur la statuaire. Certaines statues étaient célèbres pour leur beauté et le nom de leurs sculpteurs demeurait dans la mémoire collective. Athénagore rappelle le nom des sculpteurs célèbres, auteurs des statues vénérées à Éphèse, Athènes ou Délos ${ }^{11}$. Clément d'Alexandrie fournit toute une série d'exemples de statues reconnues comme des chefs-d'œuvre de Phidias: le Zeus d’Olympie et l'Athéna Polias chryséléphantine, le Zeus et l'Apollon de Patares, en Lycie... ${ }^{12}$

La beauté et le réalisme des statues ne constituaient pas les seules raisons de leur identification avec la divinité. La confusion entre la statue et la divinité était accentuée par des artifices qui permettaient de faire se mouvoir les statues. Les statues donnaient alors l'impression d'être vivantes (fig. 2). On trouve dans la littérature chrétienne une dénonciation de ces artifices permettant de donner une apparence de vie à ce qui est de la matière inerte. Plusieurs auteurs soulignent une fraude orchestrée par les prêtres des sanctuaires égyptiens dans lesquels l'on trouvait des statues articulées, ce qui permettait aux prêtres de les mouvoir, et des statues creuses laissant croire aux dévots que la divinité parlait à travers 
elles ${ }^{13}$. Théodoret de Cyr ( $\mathrm{V}^{\mathrm{e}}$ siècle) dénonce la supercherie des prêtres: «Ils fabriquaient leurs statues de bronze ou de bois avec un vide à l'intérieur et les appliquaient le dos au mur, en ménageant dans le mur des ouvertures invisibles; ensuite, ils montaient par des passages secrets et, une fois à l'intérieur des statues, ils ordonnaient à travers elles tout ce qu'ils voulaient. Les auditeurs mystifiés faisaient ce qui leur était commandé ${ }^{14}$. » Une statue d'Épicure, conservée à la Ny Carlsberg Glyptothek de Copenhague, comporte un orifice sous la lèvre inférieure et une cavité en arrière de la tête, ce qui permettait de parler à travers elle, tout en déformant assez la voix pour donner l'impression que c'était le philosophe qui discourait via sa statue ${ }^{15}$. Auteurs juifs et chrétiens partageaient la même aversion pour cette utilisation frauduleuse des statues. Rufin d'Aquilée (IV $\mathrm{V}^{\mathrm{e}} \mathrm{e}$ siècles) reprend peut-être une histoire déjà rapportée par Flavius Josèphe qui présente le scandale d'un prêtre débauché conduisant des femmes à l'adultère par le biais de la statue du dieu Saturne ${ }^{16}$.

Le mouvement des statues donnait également une impression de vie. Rufin d'Aquilée et Quodvultdeus dénoncent un mécanisme dans le Serapeum d'Alexandrie qui permettait, grâce à un aimant, d'attirer au plafond une sculpture en métal du char du Soleil, lors d'un rituel mettant en présence le Soleil et Sérapis: "L'assistance croyait que la statue s'était élevée et demeurait suspendue en l'air ${ }^{17}$.» Tous les auteurs chrétiens crient au scandale quand, par diverses méthodes, les statues semblent s'animer, ce qui fait croire à la présence de la divinité dans sa statue. Pour contrer cette idée et rassurer les nouveaux convertis, il fallait rappeler que les statues sont des objets inertes et que les dieux sont impuissants. Toutefois, la notion de statue habitée par la présence divine était si bien ancrée que l'on trouve chez d'autres auteurs chrétiens la notion que les anciens dieux, identifiés à de méchants démons, guettent les chrétiens depuis leurs statues dans l'espoir de les faire tomber dans l'idolâtrie ou de leur causer du mal.

\section{Hésitation chrétienne : inanité des statues ou présence démoniaque?}

\section{Inanité des statues}

L'inanité des idoles, l'impuissance des Baals et, par extension, celle des statues divines gréco-romaines étaient donc scandées de manière répétée aux nouveaux chrétiens convertis du paganisme, mais il leur fallait encore en intérioriser le message selon lequel les dieux ne résident ni dans un temple particulier, ni dans une statue. Les Apologistes chrétiens qui dénoncent un culte rendu à des objets inanimés peuvent s'appuyer sur les satires religieuses des philosophes qui s'étaient montrés critiques à l'égard de ce culte ${ }^{18}$, la nature spirituelle des dieux ne pouvant s'accommoder du monde matériel dans lequel voulaient les enfermer les tenants des cultes. Ainsi, Porphyre (234-ca 305), un philosophe très souvent cité par les auteurs chrétiens, écrit-il: «Le premier dieu est incorporel, immobile et indivisible; il n'est ni contenu dans quelque chose ni enfermé en lui-même, et il n'a besoin, comme on l'a dit, de rien qui vienne de l'extérieur ${ }^{19}$. »

Rendre un culte à des objets est donc contraire au bon sens, expliquent les philosophes. Celse avait cité Héraclite disant: «S'approcher des objets inanimés comme s'ils étaient des dieux, c'est faire comme si l'on bavardait avec des maisons ${ }^{20}$. " Dans le Protreptique, Clément d'Alexandrie souligne que «c'est pure niaiserie de supplier ainsi habituellement des objets insensibles, "œuvre de la main des hommes" (Ps. 115, 4) ${ }^{21}$ ». Les païens croient parler à un dieu quand ils ne s'adressent en fait qu'à des formes de marbre ou de bronze. Minucius Felix écrit des lignes ironiques sur la perspicacité des animaux: «Combien les animaux muets portent d'instinct sur vos dieux un jugement plus vrai! Les souris, les hirondelles, les milans savent parfaitement qu'ils sont insensibles: ils les foulent de leurs pattes, s'installent sur eux et, si on ne les chasse pas, font leur nid dans la bouche même de votre dieu; quant aux araignées, elles enveloppent son visage de leur toile et suspendent leur fils à sa tête même. Vous, vous les essuyez, vous les nettoyez, vous les raclez (vos tergetis, mundatis, eraditis), et ces dieux, qui sont faits par vous, vous les protégez et les redoutez ${ }^{22}$.»

L'insistance des auteurs chrétiens à souligner l'inanité des statues - chacun y consacre quelques pages - est largement liée à cette éducation qui porte à voir dans la statuaire plus que du bois, du métal ou du marbre façonné avec art. Les statues étaient investies de pouvoir et il fallait aux chrétiens toute la force de leur foi pour se convaincre profondément qu'elles n'en avaient pas. Augustin répond ainsi à Publicola, un chrétien inquiet de la contamination spirituelle créée par les sacrifices offerts aux statues divines. Cet esprit inquiet ou soucieux de bien faire reprend des questions qui sont également clairement formulées dans la littérature juive, la Mishna et le Talmud ${ }^{23}$. Comme les rabbins les plus stricts, il pense que le contact avec le monde des idoles entraîne une forme d'impureté, de souillure et de pollution. Publicola se demande par exemple s'il peut en tant que chrétien continuer à se rendre dans les thermes alors que des sacrifices (de fleurs probablement) sont offerts devant les statues. Gamaliel lui aurait répondu que si c'est un acte d'idolâtrie d'aller dans les lieux où les sacrifices ont lieu, ce n'en est pas un de se rendre dans les bains si les statues présentes en ce lieu servent d'ornement ${ }^{24}$. Augustin lui fait une réponse assez similaire, soulignant qu'il vaut mieux s'abstenir de ce qui a été offert en sacrifice, mais qu'il est inutile de s'abstenir de l'air, de l'eau et des aliments par crainte qu'ils n'aient été en contact avec un sacrifice ${ }^{25}$. Publicola était simplement trop scrupuleux, mais on trouve aussi des témoignages de peur qui montrent que vers la fin de la période antique, on commence dans certains milieux à percevoir les statues divines comme une menace. Dans la Vie de Sévère par son ami Zacharie le Scholastique ( $\mathrm{V}^{\mathrm{e}}-\mathrm{VI}^{\mathrm{e}}$ siècles), un récit montre la crainte que pouvaient inspirer les statues auxquelles des sacrifices étaient offerts. Après la découverte d'un sanctuaire clandestin à Ménouthis, non loin d'Alexandrie, pour éviter que les païens ne viennent récupérer leurs précieuses statues pour les cacher 
ailleurs, des moines et des étudiants chrétiens, envoyés par Pierre Monge, l'évêque d'Alexandrie, décidèrent non sans inquiétude de passer la nuit en compagnie des idoles. Abandonnés par le prêtre local lui aussi saisi de peur, ils chantèrent des psaumes pour se rassurer: «Leurs idoles sont d'argent et d'or, et faites de main d'hommes. Elles ont des bouches et ne peuvent parler.» (Ps. 115) Ou bien : «Les dieux des nations sont des démons mais le Seigneur a créé le ciel.» (Ps. 96) ${ }^{26}$ Les païens dépités faisaient circuler le bruit que les moines ne passeraient pas la nuit vivants en raison de leur profanation.

La même peur est exprimée au VI ${ }^{\mathrm{e}}$ siècle par des moines du mont Cassin, au moment où, au cours de travaux de construction sur le site de l'ancien temple où ils avaient choisi d'établir leur monastère, ils mirent au jour une idole de bronze. Ils paniquèrent et s'attendirent à quelque chose de terrible, attribuant finalement le décès d'un jeune moine écrasé par l'écroulement d'un mur à l'action de démon de la statue ${ }^{27}$.

C'est l'influence croissante de ces récits qui finit par modifier l'attitude de la population à l'égard de la statuaire et qui renforce finalement les croyances en ce pouvoir qu'auraient les statues d'abriter des démons. Les anciens dieux pouvaient venir d'eux-mêmes ou bien être manipulés par des rituels d'enchantement, selon une ancienne forme de magie, la telestike, un rituel d'animation des statues.

\section{L'animation des statues}

La peur des statues était liée à la croyance que non seulement les démons pouvaient prendre possession de leurs statues, mais qu'ils s'en servaient également pour observer les hommes dans le but de les conduire à l'idolâtrie ou de leur causer du tort. Les statues des dieux et des empereurs divinisés fournissaient en quelque sorte un corps aux démons, tandis que les temples leur offraient une résidence. Cette idée était très répandue, tant dans la culture religieuse populaire que dans la culture philosophico-religieuse des adeptes de la théurgie ${ }^{28}$. Les dieux venaient parfois d'eux-mêmes séjourner dans leurs statues, attirés par la vénération des fidèles et par les fumets des sacrifices ${ }^{29}$. Firmicus Maternus (IV ${ }^{\mathrm{e}}$ siècle) explique que «les esprits impurs des démons se rassemblent dans la statue elle-même comme dans les autres statues à la suite des sacrifices continuels ${ }^{30}$ ». Il fait écho à Minucius Felix, qui était convaincu que «ces esprits impurs, ces démons, comme l'ont montré les mages, les philosophes et entre autres Platon -se cachent derrière les statues et les images sacrées ${ }^{31}$ ».

Ces auteurs chrétiens de la fin de l'Antiquité connaissaient sans doute les traditions des philosophes qui, à la suite de Jamblique, pensaient que les dieux se laissent saisir pour peu qu'on leur prépare un réceptacle adapté à chacun ${ }^{32}$. Hermès Trismégiste s'était ainsi taillé la réputation d'avoir enchanté les statues. Le Corpus Hermeticum présente les statues divines comme «pourvues d'une âme, conscientes, pleines de souffle vital, et qui accomplissent une infinité de merveilles; des statues qui connaissent l'avenir et le prédisent par les sorts, l'inspiration prophétique, les songes et bien d'autres méthodes ${ }^{33}$ ». À Constantinople, c'est autour de la figure d'Apollonios de Tyane que se concentrent les légendes d'enchantement des statues. Selon Jean Malalas, Apollonios serait venu à Constantinople contrôler les animaux nuisibles grâce à leur représentation sous forme statuaire et il aurait enchanté les statues ${ }^{34}$. Apollonios était très célèbre durant l'Antiquité tardive et encore plus peut-être à l'époque byzantine où on lui attribue un traité, Apotelesmata ${ }^{35}$. Culture religieuse populaire et culture savante des lettrés se confortaient donc pour accorder aux statues un pouvoir potentiel. Tout un courant de la philosophie néoplatonicienne avait évolué vers la théurgie à la fin de l'Antiquité et commentait les pratiques magiques permettant de faire venir l'âme d'une divinité dans sa statue. Les auteurs chrétiens étaient conscients de l'existence de ces rituels paiens d'invocation de la divinité pour qu'elle vienne résider en sa statue et leur accordaient plus ou moins de crédit ${ }^{36}$. Loin de nier l'existence des divinités traditionnelles du polythéisme, la littérature chrétienne hagiographique voit en elles des êtres spirituels néfastes, des démons. Cette littérature loue donc les saints chrétiens qui s'attaquent aux idoles et les brisent.

\section{De la destruction à la valorisation comme patrimoine}

Dans certaines Vies de saints, la destruction des statues païennes est présentée comme un geste contribuant à la réputation du saint (fig. 1). Jean d'Éphèse, par exemple, rapporte l'action des saints monophysites en Orient: «Chacun en son temps courait avec zèle et énergie, fort dans sa volonté d'abolir le paganisme et de détruire l'idolâtrie, de renverser les autels et détruire les sanctuaires, ou de couper les arbres dans un zèle religieux ardent ${ }^{37} \ldots$.. On a alors l'impression d'une recherche active des lieux de résidence des démons, dans le but de libérer le monde (fig. 3). En Égypte, Bésa vante les expéditions clandestines de Shenouté, l'abbé du Monastère Blanc, contre les statues présentes dans la riche demeure d'un homme qu'il estimait être un crypto-paien. Bésa note l'enthousiasme avec lequel il entreprit sa tâche de «les briser les unes contre les autres ${ }^{38}$.» Il devenait dangereux de posséder des collections de statues comme l'apprit Gèsios, ce gouverneur de Thébaïde ${ }^{39}$, retiré sur ses terres dans la région de Panopolis qui fut probablement dénoncé par quelqu'un de sa maison ${ }^{40}$. Gèsios tenta de convaincre le terrible Shenouté de sa bonne foi, de son respect des lois et de sa conversion au christianisme en l'invitant à venir voir qu'il n'y avait pas dans sa maison d'idoles ou d'autels suspects. Shenouté constata en effet l'absence de tout objet compromettant, mais des informateurs lui indiquèrent qu'une collection de statues divines était cachée dans une pièce au second étage de la maison et une seconde expédition, s'apparentant à un cambriolage nocturne, la lui livra. Ces «idoles démoniaques» devant lesquelles des lampes avaient été allumées ${ }^{41}$ avaient été mises à l'abri par Gèsios et provenaient d'un temple mis à mal par une attaque 
de Shenouté ${ }^{42}$. Ce dernier tenait la preuve de la conversion de façade de Gèsios. Il devenait plus difficile de conserver des objets compromettants et le réflexe fut donc, chez beaucoup de dévots des anciens cultes, de cacher les statues, en la plaçant dans des pièces secrètes, des grottes, ou encore dans des puits, pour les protéger.

Il est notable que la majorité des collections de statues antiques, divines ou impériales qui nous sont parvenues l'ont été en raison de leur enfouissement volontaire. Parmi elles, on a pu retrouver de magnifiques statues, bien conservées, tout comme on a pu constater, sur des statues mutilées, les traces archéologiques du mauvais traitement subi. Certaines collections de statues ont été tout simplement mises au rebut sans autre effort que de s'en débarrasser: c'est le cas d'une collection à Antioche ${ }^{43}$. Inversement, d'autres statues ont été mutilées. Une statue d'Allat Athena, désormais au musée de Palmyre, a été retrouvée en morceaux. La tête séparée du tronc, les bras coupés, les organes des sens mutilés ${ }^{44}$. On a donc, outre des statues endommagées par leur chute ou par le temps, d'un côté des statues délibérément mutilées avant leur mise au rebut et, d'un autre côté, des statues soigneusement dissimulées dans un souci de préservation. Toute une gamme d'attitudes possibles face à la statuaire peut se déduire de ces dépôts. Le phénomène est donc complexe ${ }^{45}$.

Il faut tenir compte de la chronologie. Tandis qu'au $\mathrm{V}^{\mathrm{e}}$ siècle, des aristocrates ont pu profiter de la fermeture des temples pour se constituer à moindre frais une collection privée de belles statues, il n'était plus séant, à la fin du VI ${ }^{\mathrm{e}}$ et au VII ${ }^{\mathrm{e}}$ siècle, de posséder de tels objets dans sa maison. Un membre de la cour de Théodose II avait fait venir, pour sa propre collection, la statue de Zeus par Phidias en provenance d'Olympie, ainsi qu'une statue d'Aphrodite par Praxitèle venant de Cnide. Les empereurs eux-mêmes, jusqu'à Justinien, eurent recours aux temples pour embellir encore et toujours leur capitale. Il y avait donc à Constantinople aux $\mathrm{V}^{\mathrm{e}}$ et $\mathrm{VI}^{\mathrm{e}}$ siècles plusieurs collections de statues antiques dont un certain nombre provenait sans doute des temples désormais fermés. Elles disparurent pour beaucoup lors des incendies de la ville, en 475 et en 532, comme celle des bains de Zeuxippe ${ }^{46}$. Les élites des provinces ne furent pas en reste pour embellir leurs demeures, comme le prouvent les collections de statues découvertes à Chiragan ou dans une maison proche d'Antioche. Mais dans les deux cas, les collections ont été enfouies: il fallait se débarrasser des statues.

Ce grand ménage des statues se produit particulièrement entre les $\mathrm{V}^{\mathrm{e}}$ et $\mathrm{VII}^{\mathrm{e}}$ siècles. Certains exemples donnent à penser qu'une maison a changé de propriétaire ou que les héritiers ont changé de religion. Ainsi, en Grèce, à Athènes, on constate un nettoyage de ce qui restait de païen dans l'une des maisons de l'Aréopage qui ont été fouillées : retrait de certaines mosaïques, humiliation d'une statue d'Athéna retournée et placée de façon à ce qu'elle se fasse piétiner, décapitation des têtes de Dionysos et des nymphes sur un bas-relief. La pratique consistant à enfouir une statue face contre terre sous un mur ou un seuil est attestée plus d'une fois. Un tel procédé a été utilisé, par exemple, lors de la conversion du temple

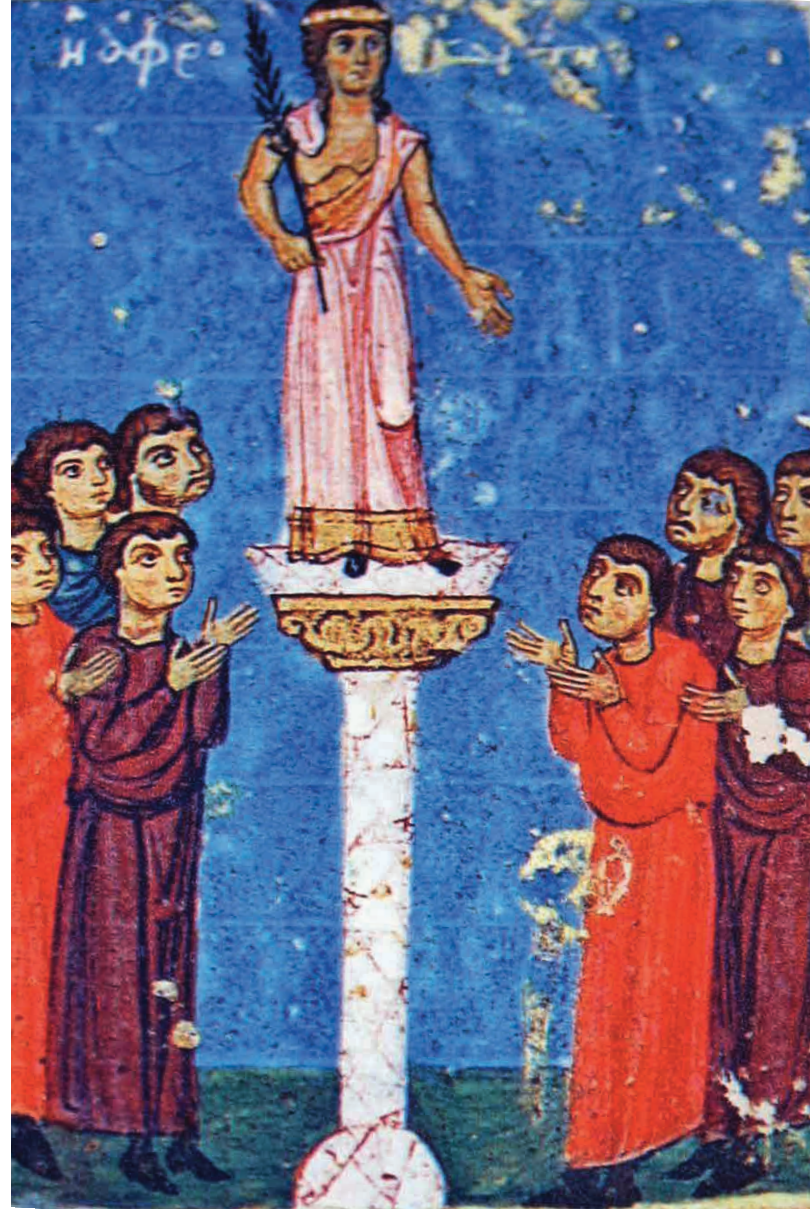

Fig. 2. Aphrodite, manuscrit

du Mont Athos, codex 6 de

Saint Pantéleimon, parchemin,

fin du IX ${ }^{\mathrm{e}}$ siècle.

(C) DR.

d'Aphrodite à Aphrodisias vers le milieu du $\mathrm{V}^{\mathrm{e}}$ siècle $^{47}$. On constate alors une véritable humiliation de la statue, traitée comme une idole vaincue et non comme une simple sculpture.

Aphrodisias a révélé une maison dans laquelle s'est joué un scénario assez proche: un groupe de statues représentant des philosophes ornait, au $\mathrm{V}^{\mathrm{e}}$ siècle, un espace qui servait peut-être d'école philosophique. Devenus importuns au $\mathrm{VI}^{\mathrm{e}}$ siècle, les portraits de philosophes ont été descellés et jetés en différents endroits, après avoir été décapités et brisés en plusieurs morceaux. Les statues divines n'étaient donc pas les seules à subir mutilation et destruction. On constate un rejet plus large de la statuaire ${ }^{48}$.

Dans la mesure où les statues ornementales pouvaient recevoir des sacrifices, un soupçon de pratiques illicites pouvait naître de leur simple présence. C'est sans doute ce qui explique la mise au rebut de plusieurs collections de statues retrouvées enfouies ou jetées dans des puits. À Athènes, un groupe de maisons bâties au IV ${ }^{\mathrm{e}}$ siècle sur l'Aréopage et abandonnées dans les années 530-550 a livré, lors des fouilles, de nombreuses statues parmi lesquelles se trouvaient un portrait d'Antonin 
le Pieux, une statue d'Hercule, une autre d'Hermès, les têtes de Némésis et d'Hélios, une Nike, mais aussi une statuette de philosophe assis, celle d'un homme barbu et deux statues de femmes. C'est l'ensemble des sculptures qui a donc été l'objet d'un rejet ${ }^{49}$.

À Scythopolis (actuellement Beth Shean, Israël), lors de l'abandon du bain oriental, daté des années 515/516, l'hypocauste fut rempli de statues. De nombreux fragments provenant de statues de différente taille y ont été retrouvés, le plus souvent sans tête. D'autres statues ont été découvertes sous le sol d'une nouvelle construction entreprise sur les lieux, la salle dite de Silvanus, parmi lesquelles une statue de Dionysos grandeur nature dont les yeux, le nez et la bouche avaient été mutilés, et une statue d'empereur dont seul le torse fut mis au jour. Le portrait de la gorgone Méduse qui ornait sa cuirasse avait été buriné. On a donc décidé de se débarrasser de tout un décor, non sans avoir pris la précaution d'empêcher la divinité représentée de pouvoir utiliser sa statue pour nuire: l'attaque des yeux, des oreilles, des mains et des pieds vise à neutraliser la capacité des démons à utiliser l'objet.

Le phénomène s'amplifie avec la crise urbaine et les invasions qui détruisent nombre de cités antiques. La statuaire ne se relève pas de pareils désastres, et achève sa vie dans des fours à chaux, en vue de permettre d'autres constructions. Le phénomène a été davantage étudié pour le monde byzantin que pour le monde musulman. Il y a toutefois un exemple qui montre que christianisme et islam pouvaient partager le même rejet de la statuaire.

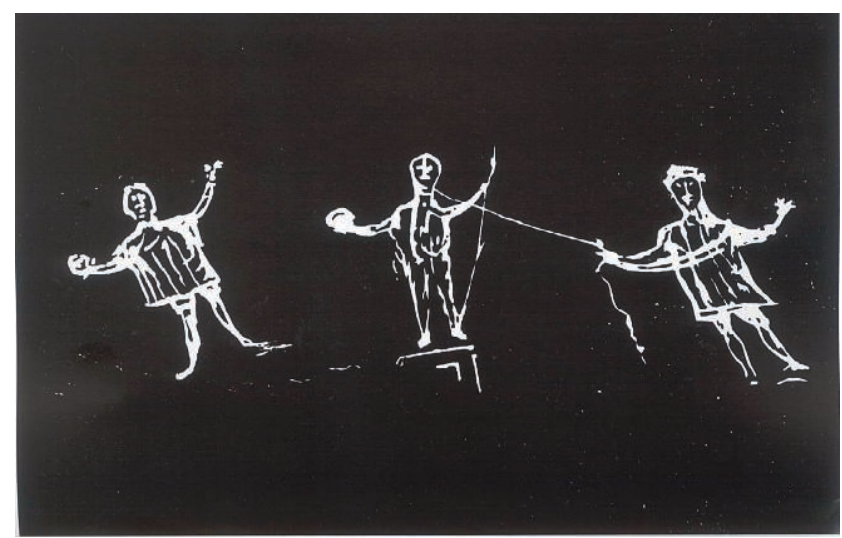

Fig. 3. Graffito, peinture sur plâtre, hypogée de la via Paisiello, Rome: statue détruite par jet de pierre et abattue par une corde, datant peut-être de la fin du IV ${ }^{\mathrm{e}}$ siècle. (C) DR.
Parmi les trouvailles de grandes collections de statues enfouies, on peut signaler les 245 fragments d'un groupe de 28 sculptures de divinités ou de figures mythologiques en marbre datant du $\mathrm{I}^{\mathrm{er}}$ au $\mathrm{IV}^{\mathrm{e}}$ siècle qui ont été retrouvées au cours de fouilles du sanctuaire de Pan à Césarée Philippi, à la frontière de la Palestine et de la Syrie ${ }^{50}$. Ce sanctuaire très important, dominant la cité avec ses multiples temples, a été abandonné vers le milieu du $\mathrm{V}^{\mathrm{e}}$ siècle, mais les statues ont été retrouvées dans l'une des salles ainsi que dans la rue, devant le bâtiment, dans une couche du début de l'époque musulmane. Elles proviennent probablement de ce grand sanctuaire qui comportait plusieurs temples. Les statues ont donc été regroupées, peut-être enfermées dans le vestibule, ce qui correspond à ce que recommande alors la législation. Au final, elles ont été enterrées en même temps que des blocs de construction et de la terre. Il semble important de souligner que cet enfouissement a eu lieu loin du temps des conflits entre païens et chrétiens, dans un contexte différent, celui d'un changement de décor, qui touche non seulement l'espace public, mais aussi les espaces privés et les espaces religieux. Si l'enfouissement s'est opéré entre la fin du VII ${ }^{\mathrm{e}}$ et le IX ${ }^{\mathrm{e}}$ siècles, il est intéressant de le relier à la période d'iconoclasme, ce que S. Fine a étudié pour les synagogues et R. Schick pour les églises ${ }^{51}$ : une période au cours de laquelle on constate un nettoyage des éléments du décor ne correspondant plus aux normes du jour. L'influence musulmane est possible. Le décor autrefois acceptable, qui incluait des représentations d'Hélios, des signes du zodiaque ainsi que des figures animales fut rejeté au profit d'un décor plus aniconique.

Finalement, les statues antiques disparaissent les unes après les autres au début du Moyen Âge. Seules demeurent celles qui sont protégées par les empereurs byzantins, ou par une mauvaise identification. La célèbre statue de Marc-Aurèle, au Capitole à Rome, ne doit sa survie qu'au fait qu'on la prenait pour une statue de Constantin au début du Moyen Âge. Il fallut plusieurs siècles pour qu'une valorisation de ce qui restait du patrimoine antique ne soit effective. Après le pillage de l'hippodrome par les Latins de la quatrième croisade, en 1204, Nikétas Choniatès, un auteur byzantin, se lamente de la perte de ces chefs-d'œuvre antiques, qui ont été fondus pour en faire de la monnaie, ou bien transportés à Venise et dans d'autres centres urbains d'Occident. Pour les lettrés byzantins, ces œuvres que leurs ancêtres regardaient avec méfiance encore durant le haut Moyen Âge, faisaient désormais partie du patrimoine et pouvaient être regrettées ${ }^{52}$.

\section{Notes}

1. En 399, un texte posté à Padoue prévoit que les idoles des temples, qui, malgré l'interdiction absolue de sacrifices, reçoivent un culte seront déposées et placées sous le contrôle des autorités impériales: Codex Theodosianus, 16, 10, 18; un texte similaire fut posté à Rome en 407 pour que les statues des temples qui continuent à recevoir un culte soient arrachées de leur socle: Codex Theodosianus, 16, 10, 19.
2. Codex Theodosianus, 16, 10, 8 .

3. Clément d'Alexandrie, Protreptique, trad. Cl. Mondésert, Paris, 1941, IV, 57, p. 112; éd. M. Marcovich, Clementis Alexandrini Protrepticus (Supplements to Vigiliae Christianae, XXXIV), Leyde, 1995.

4. Clément d'Alexandrie, Protreptique, IV, 48, op. cit., p. 100.

5. Minucius Felix, Octavius, XXIV, 5, texte établi et traduit par J. Beaujeu, Paris, 1964, p. 38 (CUF) : «Mens imperitorum artis concinnitate decipitur, auri fulgore praesttingitur, argenti nitore et candore eboris hebetatur. »
6. Théophile d'Antioche, Trois livres à Autolycus, II, 2, trad. J. Sender, introduction et notes par G. Bardy, Paris, 1948, p. 76, (SC 20).

7. Minucius Felix, Octavius, XXIV, 6, op. cit., p. 38.

8. Origène, Contre Celse, I, 5 , éd. et trad. M. Borret, Paris, 1967, p. 88-89, (SC 132).

9. Tertullien, Apologétique, XII, 6, texte établi par J.-P. Waltzing, Paris, 1929, p. 70-71, (CUF). 
10. Minucius Felix, Octavius, XXIV, 7, op. cit., p. 39.

11. Athénagore, Supplique au sujet des chrétiens, XVII, 4, introduction, texte et traduction par B. Pouderon, Paris, 1992, p. 125 .

12. Clément d'Alexandrie, Protreptique, IV, 47, introduction, traduction et notes par Cl. Mondésert, Paris, 1949, p. 97.

13. G. Maspéro, «Les statues parlantes de l'Égypte antique ", Causeries d'Égypte, Paris, 1907, p. 167-173; F. Thélamon, Païens et chrétiens au IV siècle. L'apport de l'Histoire ecclésiastique de Rufin d'Aquilée, Paris, 1981, p. 240-243.

14. Théodoret de Cyr, Histoire ecclésiastique, V, 22, éd. L. Parmentier, Theodoret Kirchengeschichte, rev. G. Ch. Hansen, Berlin, 1998, p. 320 ; trad. P. Canivet revue et annotée par J. Bouffartigue et alii, Théodoret de Cyr, Histoire ecclésiastique, Livres III-V, Paris, 2009, p. 432-435.

15. F. Poulsen, "Talking, Weeping and Bleeding Sculptures”, Acta Archaeologica, 16, 1945 , p. 178-195.

16. Rufin, Histoire ecclésiastique, II, 22 éd. Th. Mommsen, Leipzig, 1903-1909, p. 1027, (GCS 9, 2).

17. Rufin, Histoire ecclésiastique, II, 23 , éd. Th. Mommsen, Leipzig, 1903-1909, p. 1028 (GCS 9, 2) ; trad. F. Thélamon, Païens et chrétiens au IV siècle. L'apport de l'Histoire ecclésiastique de Rufin d'Aquilée, Paris, 1981, p. 182; Quodvultdeus, Liber promissionum, III, 42, éd. et trad. R. Braun, Paris, 1964, p. $570-71$.

18. H.W. Attridge, "The Philosophical Critique of Religion under the Early Empire", ANRW, 16:1, Berlin, 1978, p. 45-78. 19. Porphyre, De abstinentia, II, 37, éd. et trad. J. Bouffartigue, M. Patillon, De l'abstinence, Paris, 1977, t. II, p. 103.

20. Origène, Contre Celse, I, 5 , introduction, texte critique, traduction et notes par M. Borret, Paris, 1967, p. 88-89.

21. Clément d'Alexandrie, Protreptique, IV, 46, op. cit., p. 97 ; éd. M. Marcovich, Clementis Alexandrini Protrepticus (Supplements to Vigiliae Christianae, XXXIV), Leyde: Brill, 1995 , p. 71.

22. Minucius Felix, Octavius, XXIV, 9, op. cit., p. 39.

23. Tosefta, II, 5-6

24. Mishna, Avodah Zarah 3. 4.

25. Augustin, ep. 47, CSEL 34, 2, p. 129-136.

26. Zacharie le Scholastique, Vie de Sévère, éd. M. A. Kugener, P.O., 2, p. 27-32. Sur cet épisode daté du milieu du $\mathrm{V}^{\mathrm{e}}$ siècle: E. Wipscyska, «La christianisation de l'Égypte aux $\mathrm{IV}^{\mathrm{e}}-\mathrm{VI}^{\mathrm{e}}$ siècles. Aspects sociaux et ethniques", in Aegyptus, 68, 1988, p. 117164; Ch. Haas, Alexandria in Late Antiquity. Topography and Social Conflict, Baltimore, 1997. $1-2$.
27. Grégoire le Grand, Dialogues, II, 10,
28. H. Chadwick, "The Philosophical Tradition and the Self”, Late Antiquity. A Guide to the Postclassical World, éd. G. Bowersock, P. Brown, O. Grabar, Cambridge, 1999, p. 71; G. Fowden, The Egyptian Hermes. A Historical Approach to the Late Pagan Mind, Princeton, 1993.

29. J.-P. Mahé, Hermès en Haute-Égypte, t. II : Le fragment du Discours Parfait et les Définitions Hermétiques arméniennes (NH VI, 8.8a), Québec: Les Presses de l’Université Laval, 1982, p. 957102; F. Heim, «L'animation des statues chez les Apologistes du $\mathrm{III}^{\mathrm{e}}$ siècle ", Culture antique et fanatisme. Textes réunis par J. Dion, Nancy, 1996, p. 95-102.

30. Firmicus Maternus, De errore profanarum religionum, XIII, 4, op. cit., p. 106

31. Minucius Felix, Octavius, XXVII, 1 op. cit., p. 45-46

32. Jamblique, Les mystères d'Égypte, V, 23, éd. et trad. E. des Places, Paris, 2003, p. $178-179$.

33. Hermès Trismégiste, Asclépius, 24, trad. A.-J. Festugière, dans Hermès Trismégiste, Corpus Hermeticum. Traités XIII-XVIII, Paris, 2008 , p. 326.

34. Jean Malalas, Chronographia, X, 51, éd. H. Thurn, Berlin, 2000, p. 199-200. (CFHB 35) ; W. L. Dulière, «Protection permanente contre les animaux nuisibles assurée par Apollonius de Tyane ", Byzantinische Zeitschrift, 63, 1970, p. 247-277.

35. F. Nau, «Apotelesmata Apollonii Tyanensis », Patrologia Syriaca, 1, 2, Paris, 1907, p. 1363-1392. Ch. Jones le date de l'époque byzantine (après 800) : Ch. Jones, "Apollonius of Tyana in Late Antiquity", Greek Literature in Late Antiquity. Dynamism, Didacticism, Classicism, éd. S. F. Johnson, Aldershot, 2006, p. 49-64.

36. F. Heim, «L'animation des statues chez les Apologistes ", in Culture antique et fanatisme, éd. J. Dion, Nancy, 1996, p. 95-102.

37. Jean d'Éphèse, History of the four excellent deacons, earnest in religion, Abraham, Cyriac, Barhadbshabba, and Sergius, in Lives of the Eastern Saints, PO 17, Paris, 1923, p. 658-660.

38. Besa, Life of Shenoute, trans. by David N. Bell, Kalamazoo, 1983, p. 66.

39. L'identité du personnage dénoncé par Shenouté a fait l'objet de nombreuses recherches, S. Emmel, "From the Other Side of the Nile: Shenute and Panopolis", Perspectives on Panopolis. An Egyptian Town from Alexander the Great to the Arab Conquest, éds. A. Egberts, B. P. Muhs, J. Van der Vliet, Leyde, 2002, 95-113; H. Behlmer, "Historical Evidence from Shenoute's De extremo Judicio", Sesto Congresso Internazionale di Egittologia. Atti, G. M. Zaccone, T. R. di Netro (éds), Turin, 1993, t. 2, 11-19; dossier de textes dans H. J. Thissen, "Zur Begegnung von Christentum und 'Heidentum'. Shenoute und Gessios”, Enchoria, 19-20 (1992-1993), p. 155-164.
40. S. Emmel, "Shenoute of Atripe and the Christian Destruction of Temples in Egypt: Rhetoric and Reality", From Temple to Church. Destruction and Renewal of Local Cultic Topography in Late Antiquity, éds. J. Hahn, S. Emmel, U. Gotter, Leyde 2008, p. 161-201; id., "From the Other Side of the Nile: Shenute and Panopolis", Perspectives on Panopolis. An Egyptian Town from Alexander the Great to the Arab Conquest, Leyde, 2002, p. 95-113.

41. Shenouté, "Que nos yeux", trad S. Emmel, "Shenoute of Atripe and the Christian destruction”, p. 186; attaque du temple d'Atripe commentée, p. 142-143.

42. D. Frankfurter, "Iconoclasm and Christianization in Late Antique Egypt: Christian Treatments of Space and Image", From Temple to Church., p. 135-159.

43. D. M. Brinkerhoff, A Collection of Sculpture in Classical and Early Christian Antioch, New York, 1970.

44. B. Gassowska, "Maternus Cynegius, praefectus Praetorio Orientis and the Destruction of the Allat Temple in Palmyra", in Archeologia, XXXIII, 1982, p. 107-123.

45. Sauer E., The Archaeology of Religious Hatred in the Roman and Early Medieval World, Stroud, 2003.

46. C. Mango, "Antique Statuary and the Byzantine Beholder", DOP XVII, 1963, p. 53-75.

47. R. Cormack, "Byzantine Aphrodisias. Changing the Map of a City", Proceedings of the Cambridge Philosophical Society, 214, n.s. 24, 1990, p. 26-41.

48. R.R. R. Smith, "Late Roman Philosopher Portraits from Aphrodisias", Journal of Roman Studies, 80, 1990, p. 127-155.

49. A. Frantz, The Athenian Agora, vol. XXIV, The American School of Classical Studies at Athens, Princeton 1988, 41.

50. E. A. Friedland, "Graeco-Roman Sculpture in the Levant: the Marbles from the Sanctuary of Pan at Caesarea Philippi (Banias)", The Roman and Byzantine Near East, t. 2: Some Recent Archaeological Research, éd. J. H. Humphrey, Journal of Roman Archaeology, Supplementary Series n 31, Portsmouth, 1999, p. 7-22.

51. Steven Fine, "Iconoclasm and the Art of Late-antique Palestinian Synagogues" dans From Dura to Sepphoris, eds. Lee I.

Levine and Ze'ev Weiss (Portsmouth, 2000), 183-194; R. Schick, The Christian Communities of Palestine from Byzantine to Islamic Rule (Princeton, 1995).

52. T. Papamatorakis, "Interpreting the De Signis of Niketas Choniates", Niketas Choniates. A Historian and a Writer, éd.

A. Simpson, St. Efthymiadis, Genève, 2009, p. 209-223. 